\title{
Digitale Souveränität als Trend?
}

\section{Der Werkzeugmaschinenbau als wegweisendes Modell für die deutsche Wirtschaft}

\author{
Annelie Pentenrieder ${ }^{\bowtie}$, Anastasia Bertini, und Matthias Künzel \\ Institut für Innovation und Technik (iit), Berlin, Deutschland \\ \{pentenrieder, bertini, kuenzel\} diit-berlin. de
}

\begin{abstract}
Zusammenfassung. Digitale Technologien und ihre Auswirkungen auf Arbeitsverhältnisse beschäftigen aktuell viele Branchen. Fachkräfte ebenso wie Manager können jedoch schwer einschätzen, was für sie und ihr Unternehmen - insbesondere für kleinere Unternehmen - nötig ist, um inmitten der Digitalisierung weiterhin souverän zu agieren. Digitale Souveränität bedeutet in diesem Kontext, den Überblick über neue technische Möglichkeiten zu behalten, um informiert entscheiden und zwischen alternativen digitalen Angeboten das Passende für das eigene Unternehmen auswählen zu können. Im Fokus des Beitrags stehen KMU im Werkzeugmaschinenbau, denn gerade dieser Industriezweig steht seit vielen Jahrzehnten für das Gestaltungsprinzip der Souveränität von Menschen im Umgang mit Maschinen ein. Hochqualifiziertes Fachpersonal wird in diesem Bereich stetig an hochtechnisierten Maschinen aus- und weitergebildet. Dieser Umstand führt dazu, dass im Werkzeugmaschinenbau Hightech mit hoher Flexibilität angeboten werden kann. Auf der Grundlage von Literaturrecherchen und Experteninterviews aus den Bereichen Produktionstechnik, Arbeitswissenschaften und Geschäftsmodellentwicklung wurden Themen und Trends identifiziert, die mit der Frage nach digitaler Souveränität im Zusammenhang stehen: rechtliche Rahmenbedingungen, Datenschutz, digitaler Kompetenzaufbau und -entwicklung, Datenspeicherkonzept, globaler Wettbewerb, Unternehmensgröße und damit verbundene Handlungsspielräume, Unternehmenskollaborationen, Austausch von Informationen zwischen den Unternehmen, Individualisierung von KI-basierten Technologien. Es zeigt sich, dass „digitale Souveränität” ein zukünftiges Gestaltungsprinzip digitaler Systeme werden könnte, das gerade für KMU im Werkzeugmaschinenbau innovationsfördernde Auswirkungen haben kann.
\end{abstract}

Schlüsselwörter: Digitale Souveränität · Werkzeugmaschinenbau · Transparente Technikgestaltung 


\section{Einführung}

Digitale Technologien und ihre Auswirkungen auf Arbeitsverhältnisse beschäftigen aktuell viele Branchen. Neue Ausprägungen der Digitalisierung - wie Cloudkonzepte, künstliche Intelligenz (KI) oder smarte Fabriken - lassen als Begrifflichkeiten noch unklar, was die technischen Infrastrukturen und Prozesse dahinter tatsächlich leisten können und wie mit ihnen im Arbeitsalltag umzugehen ist. Für Fachkräfte ebenso wie für die Manager:innen einzelner Unternehmen ist schwer einzuschätzen, was zukünftig erforderlich sein wird, um inmitten dieser komplexen Technologievielfalt als Mensch und als Unternehmen - insbesondere als kleines, mittelständisches Unternehmen (KMU) - souverän zu bleiben. Souverän bedeutet in diesem Kontext, einen Überblick über neue technische Möglichkeiten zu erhalten, um informiert zwischen alternativen digitalen Optionen das passende mehrwertstiftende Angebot für das eigene Unternehmen auswählen zu können. Besonders attraktiv sind dabei jene Technologien, mit denen weiterhin alle Geschäftsprozesse eigenständig bestimmt, gestaltet und verantwortet werden können. Auf Basis dieser Herausforderungen ist ein Diskurs um digitale Souveränität entstanden (BMWi 2018; Stubbe et al. 2019; Wittpahl 2017), der sich von Fragen zu Datenschutz und Datenhoheit (Ensthaler und Haase 2017) bis hin zur Erklärbarkeit von KI-Technologien (Wachter et al. 2018; Molnar 2019) erstreckt. Gegenwärtig zeichnet sich ab, dass viele digitale Technologien vor allem größeren Unternehmen einen Wettbewerbsvorteil durch Skalierung und Kostendegression ermöglichen: Während im produzierenden Bereich circa $25 \%$ der Großunternehmen KI-Technologien einsetzen, sind es bei den KMU nur $15 \%$ der Unternehmen (PAiCE 2018).

Der Werkzeugmaschinenbau bietet sich aus den folgenden Gründen als Branche an, um an ihm das Konzept der digitalen Souveränität - auch modellhaft für andere Branchen - weiter zu schärfen:

- Die hochtechnisierten Werkzeugmaschinen deutscher Unternehmen nehmen aktuell eine starke Position am Weltmarkt ein.

- Sowohl die Anbieter- als auch die Anwenderseite von Werkzeugmaschinen besteht aus einer heterogenen Mischung großer und kleiner Unternehmen.

- Speziell im Werkzeug- und Formenbau, in dem Werkzeugmaschinen im Alltag angewendet werden, können vielfältige Arbeitsprozesse hochqualifizierter Fachkräfte betrachtet werden.

- Das Zusammenspiel aus hochkomplexen Technologien und hochqualifizierten Fachkräften kann neue Ideen hervorbringen, wie digitale Souveränität auch in Zukunft gewährleistet werden kann.

Werkzeugmaschinen als langlebige Investitionsgüter setzen eine breite Vielfalt von umformenden, subtraktiven und additiven Fertigungsverfahren um. Häufig in Einzel- und Kleinserienfertigungen verwenden hochausgebildete Facharbeiter:innen bei den Anwenderunternehmen solche hochtechnisierten Arbeitsmittel wie etwa 
CNC-gesteuerte 5-Achs-Fräsmaschinen. Gerade von deutschen Herstellern wurden seit den 1980er Jahren dazu werkstattorientierte CNC-Steuerungs- und Programmiersysteme entwickelt, die den Facharbeiter:innen passende Werkzeuge für ihre Arbeit zur Verfügung stellen (Blum und Hartmann 1988). Das nutzerzentrierte und lernförderliche Technikdesign, mit dem diese Systeme und vor allem ihre Benutzeroberflächen gestaltet sind, hat erheblich dazu beigetragen, dass trotz des Einzugs von Automatisierung und Industrierobotern (3. industrielle Revolution) die Kompetenzen von Facharbeiter:innen in der Produktion erhalten und weiterentwickelt werden konnten. Mit der Digitalisierung zeichnet sich nun ab, dass durch den Einsatz von Techniken der KI sogar für die Einzel- und Kleinserienfertigung hoch anspruchsvoller Werkstücke weitere Automatisierungsschritte möglich sind (4. industrielle Revolution). Daraus ergibt sich die Herausforderung, für das stetig komplexer werdende technische Niveau Lösungen zu finden, durch die den hochqualifizierten Facharbeiter:innen weiterhin vielfältige Handlungsmöglichkeiten gegeben werden.

Im vorliegenden Beitrag werden zunächst aktuelle Trends der Digitalisierung im Werkzeugmaschinenbau identifiziert. Daraus zeichnet sich ab, dass gerade für KMU, die im Vergleich zu großen Unternehmen nur begrenzte Ressourcen für Forschung und Entwicklung aufwenden können, solche qualitativen Umbrüche herausfordernd sein können. Im zweiten Schritt werden daher zwei sich ergänzende Themenkomplexe vertieft analysiert, die insbesondere für KMU relevant sein können: Die digitale Kompetenzentwicklung von Mitarbeiter:innen (Punkt 3 in Tab. 1, näher besprochen in 2.2) und die Individualisierung KI-basierter Technologien (Punkt 9 in Tab. 1, näher besprochen in 2.3). Diese beiden Digitalisierungsthemen fokussieren sich insbesondere auf die Interaktion zwischen komplexen digitalen Technologien und Facharbeiter:innen im Shopfloor. Mit den Konzepten von „Lernförderlichkeit“ (siehe Exkurs 1) und „Erklärbarer KI“ (siehe Exkurs 2) werden daraufhin Wege aufgezeigt, die die digitale Souveränität von Facharbeiter:innen ins Zentrum rücken. Diese Gestaltungsprinzipien können insbesondere für KMU interessant sein, da hier die Souveränität der Fachkräfte einen zentralen Stellenwert einnimmt.

\section{Trends der Digitalisierung im Werkzeugmaschinenbau}

\subsection{Bestandsaufnahme}

Zur Identifizierung aktueller Trends der Digitalisierung im Werkzeugmaschinenbau wurden im Frühjahr 201916 teilstandardisierte, telefonische Interviews mit Expert:innen aus den Bereichen Produktionstechnik, Arbeitswissenschaften und Geschäftsmodellentwicklung geführt. Anschließend wurden ihre Einschätzungen um eine Literaturrecherche ergänzt und neun Themen identifiziert, die die Expert:innen sowohl als aktuelle Hemmnisse als auch als zukünftige Chancen für den Werkzeugmaschinenbau bewerteten. 
Tab. 1. Hemmnisse und Chancen aktueller Trends in der Digitalisierung

\begin{tabular}{|c|c|}
\hline Hemmnisse & Chancen \\
\hline \multicolumn{2}{|l|}{ 1. Rechtliche Rahmenbedingungen } \\
\hline $\begin{array}{l}\text { Die bestehende Gesetzeslage ist nicht voll- } \\
\text { ständig auf die Bedarfe der Digitalisierung } \\
\text { zugeschnitten. Darum ist das Tätigwerden des } \\
\text { Gesetzgebers erforderlich: Zur Beseitigung teil- } \\
\text { weise bestehender rechtsfreier Räume und der } \\
\text { damit einhergehenden Rechtsunsicherheit, die } \\
\text { innovationshemmend wirkt }\end{array}$ & $\begin{array}{l}\text { Neue rechtliche Rahmenbedingungen } \\
\text { wirken innovationsfördernd (z. B. Wett- } \\
\text { bewerbsvorteile und Innovationsanreize } \\
\text { über virtuelle Zertifizierung) }\end{array}$ \\
\hline
\end{tabular}

\section{Datenschutz}

Datenschutz kann Vernetzungsmöglichkeiten hemmen

Ein starker Datenschutz kann ein fördernder Faktor für nutzerzentrierte Software sein, die transparent aufzeigt, welche Daten wie und von wem verwendet werden

\section{Digitale Kompetenzentwicklung}

Weiterbildung wird häufig nicht als Voraussetzung für Innovation gesehen. In so einem Fall verändern sich Berufsbilder durch die Digitalisierung, ohne dass Unternehmen dies erkennen, strategisch behandeln und damit aktiv die Digitalisierung gestalten

\section{Datenspeicherkonzept}

Klassische zentralisierte Cloud-Konzepte schaffen dem Cloud-Betreiber einen großen Vorteil, da er die Nutzungsregeln bestimmt und damit die Daten aller seiner Kunden für sich nutzbar machen kann

\section{Globaler Wettbewerb}

Der Megatrend Globalisierung bewirkt, dass die internationale Ausrichtung immer wichtiger wird für KMU. Dafür sind Investitionen u. a. in die Produktionsvernetzung notwendig, die größere Unternehmen eher fähig sind zu tätigen
Kontinuierliche Weiterbildung, die eine Mitbestimmung von Mitarbeiter:innen einschließt, ermöglicht eine nachhaltige Digitalisierung und damit eine erfolgreiche Einführung und Akzeptanz von Neuerungen

Dezentrale Peer-to-Peer-Konzepte, die
aus einzelnen Edge-Clouds bestehen,
sichern den nutzerspezifischen Zugriff
auf Betriebsdaten und vereinfachen die
Implementierung von dezentralen und damit
passgenauen Nutzungsrechtekonzepten
auch auf kleinskaligen Ebenen

Deutschland könnte davon profitieren, seinen eigenen Weg zu gehen, statt sich vornehmlich an digitalen Lösungen aus China und den USA zu orientieren. Das eröffnet Potenziale für weitere Innovationen, die Deutschland von den Konkurrenten unterscheiden

\section{Unternehmensgröße und damit verbundene Handlungsspielräume}

Heutige KI-Lösungen basieren meist auf der Verfügbarkeit großer Datenmengen zum maschinellen Lernen. Aus diesem Grund sind größere Unternehmen aktuell besser dafür ausgestattet, groß angelegte Software zu nutzen (z. B. SAP) oder eigene Software und damit Digitalstrategien zu entwickeln. Diese neuen Ausprägungen der Digitalisierung stellen KMU hingegen vor Herausforderungen
Kleine Unternehmen können schneller auf veränderte Anforderungen reagieren. Spezielle KI-Methoden, die auf kleinere Datenmengen ausgerichtet sind, können Alleinstellungsmerkmale begründen und die Nutzbarkeit für KMU stärken. Bei individualisierten Digitallösungen sind Mitarbeiter:innen in einem KMU eher in der Lage die Neuerungen mitzugestalten 
Tab. 1. (Fortsetzung)

\begin{tabular}{l|l}
\hline \multicolumn{1}{c}{ Hemmnisse } & \multicolumn{1}{c}{ Chancen } \\
\hline \multicolumn{2}{l}{ 7. Unternehmenskollaborationen } \\
\hline $\begin{array}{l}\text { Eine großskalig angelegte Software passt häufig } \\
\text { nicht zu den Anforderungen der KMU, sodass } \\
\text { zahlreiche ,Insellösungen“ existieren, die nicht } \\
\text { übertragbar sind }\end{array}$ & $\begin{array}{l}\text { Maßgeschneiderte Softwareprodukte einzel- } \\
\text { ner KMU können fach- und unternehmens- } \\
\text { übergreifend wertvolle Anregungen bieten } \\
\text { und in Kollaborationen die Grundlage } \\
\text { sein, um Ansätze zu entwickeln, die über } \\
\text { existierende Angebote hinausgehen }\end{array}$ \\
\hline
\end{tabular}

\section{Informationsaustausch zwischen Unternehmen}

\begin{tabular}{|c|c|}
\hline $\begin{array}{l}\text { Aktuelle digitale Technologien sind geprägt } \\
\text { von fehlender Vernetzung zwischen Maschinen, } \\
\text { Standorten und Unternehmen. „Sprachbarrieren“ } \\
\text { und unterschiedliche Formate erzeugen zusätz- } \\
\text { liche Schwierigkeiten }\end{array}$ & $\begin{array}{l}\text { Ansätze etwa aus dem Open-Source- } \\
\text { Bereich (OPC-UA, Automation ML) bieten } \\
\text { Grundlagen zum Ausbau überregionaler } \\
\text { Strukturen zum Informationsaustausch. Vor } \\
\text { allem existiert Bedarf an Good-Practice- } \\
\text { Beispielen mit Bezug zu Digitalisierung } \\
\text { sowie KI-basierten Assistenzsystemen oder } \\
\text { Cloud-Diensten (Künzel et al. 2019. S. 13) }\end{array}$ \\
\hline
\end{tabular}

\section{Individualisierung KI-basierter Technologien}

Vor allem in KMU herrscht Unsicherheit, was sie Es besteht ein hoher Bedarf an Erprobungsim Einzelnen von KI-Technologien oder CloudDiensten erwarten können szenarien, um konkrete KI-basierte Technologien kennenzulernen und kostengünstig entlang der eigenen Anforderungen für das eigene Unternehmen auszuprobieren

Je nach Ausgestaltung werden diese Themen der Digitalisierung von den Expert:innen aktuell als ein Hemmnis für die Digitalisierung im Werkzeugmaschinenbau wahrgenommen, aber auch als eine innovationsfördernde Chance. Einerseits geraten Unternehmen hinsichtlich des globalen Wettbewerbs unter Druck, schnell zu digitalisieren und eine bestimmte Art heute gängiger Technologien einzuführen. Diese Technologien stoßen zum Teil aus unterschiedlichen Gründen auf fehlende Akzeptanz bei den Fachkräften. Andererseits bietet sich mit der aktuellen technologischen Neuaufstellung die Möglichkeit, eigene Innovationen digitaler Produkte voranzutreiben, um sich in neu entstehenden Geschäftsfeldern von Konkurrenten unterscheidbar zu machen. Hindernisse, die aktuell noch mit der Digitalisierung in Verbindung stehen, können damit zu innovationsfördernden Kräften werden. Digitalisierung als Chance oder als Hemmnisse zu begreifen steht insbesondere für KMU, aber auch für hochqualifizierte Fachkräfte mit der Frage in Zusammenhang, wie ,digital souverän“ der Umgang mit digitalen Technologien gestaltet wird. Gerade für eine passgenaue Digitalisierung für den Mittelstand könnte die digitale Souveränität als Gestaltungsprinzip einen großen Vorteil bieten (siehe Tab. 1).

Im Folgenden werden zwei der oben genannten Trends genauer betrachtet: Die digitale Kompetenzentwicklung von Fachkräften (Punkt 3) und die Individualisierung KI-basierter Technologien (Punkt 9). Beide Themen haben gemein, dass sie die digitale Souveränität hochqualifizierter Fachkräfte an komplexen Maschinen betreffen. Im Folgenden werden die Erkenntnisse aus den Experteninterviews darum 
mit weiteren Studien und Überlegungen zur lernförderlichen Arbeitsgestaltung und der Erklärbarkeit komplexer Systeme angereichert. Die weiteren, in der Tabelle genannten Themenbereiche werden hier keiner genaueren Analyse unterzogen, da sie eher auf der Unternehmensebene angesiedelt sind.

\subsection{Digitale Kompetenzentwicklung}

Inwieweit digitale Technologien Mehrwert für ein Unternehmen erbringen, hängt davon ab, wie sie in den Arbeitsprozess von Fachkräften eingebunden werden. Vor allem bei kleinen Unternehmen besteht die Sorge, dass die Digitalisierung standardisierte und unflexible Automatisierungen mit sich bringt, die die Gestaltungsfreiheit von Fachkräften behindern, während die Technologien zudem teuer im Unterhalt sind. Dies könnte Innovationen seitens der Mitarbeiter:innen hemmen, die sonst im Shopfloor stattfinden. Gerade die Handlungs- und Gestaltungsfreiheit der Fachkräfte hängt davon ab, welche Bedienkonzepte implementiert werden. Aktuell werden diese häufig ohne Partizipation der Anwendenden programmiert. Eine fern von den Anwendenden vorgenommene Technikgestaltung identifizierten die befragten Expert:innen als Hindernis der Digitalisierung. Verstärkt wird dies, wenn die Bedienung der Maschinen und Anlagen auf produktionsbedingte oder standortbedingte Erfahrungswerte von Fachkräften angewiesen ist. Eine externe und standortferne Programmierung digitaler Unterstützungs- und Bedienkonzepte kann die spezifischen Faktoren vor Ort nicht ausreichend berücksichtigen, wenn Fachkräfte nicht bereits vorab in die Technikgestaltung miteinbezogen werden. Diese klassische Trennung von Technikgestaltung (Engineering) und Techniknutzung (Shopfloor) birgt die Gefahr, dass Bediener:innen ihr für die Fertigung zentrales Know-How weniger in den Produktionsprozess miteinbeziehen können: „Wenn jeder Schritt der Montage vom digitalen Assistenzsystem vorgegeben wird, kann man da bald auch eine Anlernkraft hinstellen, wo heute ein Facharbeiter ist", so ein Befragter der Studie von Jürgen Dispan und Martin Schwarz-Kocher (Dispan und Schwarz-Kocher 2019, S. 7).

Wird jedoch eine informierte Fachkraft von Beginn an in der Technikgestaltung mitgedacht, können Fachkräfte souverän die Grenzen digitaler Technologien kennenlernen, Konsequenzen etwa algorithmisch empfohlener Entscheidungen (z. B. KI) einschätzen und gegebenenfalls sogar individuell an situative Besonderheiten im Shopfloor anpassen. Dazu benötigen die Anwendenden jedoch ein fundiertes Verständnis der neuen digitalen Technologien. Zur Etablierung solcher digitalen Kompetenzen sind Konzepte erforderlich, die auf zwei Seiten ansetzen: zum einen bei Schulungen des Personals und zum anderen beim Design von Benutzerschnittstellen für digitale Technologien. An beiden Domänen kann die digitale Souveränität der Fachkräfte gefördert werden. Nur wenn die Informationen digitaler Systeme für die Fachkraft verständlich sind, kann beispielsweise auch auf seltene Fehlermeldungen angemessen reagiert werden. Für den aktiven Ausbau digitaler Kenntnisse von Fachkräften müssen Unternehmen flexible Weiterbildungsmöglichkeiten in den Arbeitsalltag integrieren. Lernförderliche Arbeitsplätze erlauben ein eigenständiges Lernen auch im Alltagsgeschäft und schaffen die in der Arbeitsorganisation dafür notwendigen zeitlichen und räumlichen Freiheiten für Fachkräfte (vgl. Gestaltungsprinzip Lernförderlichkeit). 


\section{Exkurs 1: Gestaltungsprinzip Lernförderlichkeit}

Lernförderlichkeit ist ein Begriff aus den 1980er Jahren, der im Rahmen des Programms „Humanisierung von Arbeit“ entwickelt wurde und für heutige digitale Lernumgebungen wieder relevant wird. Die lernförderliche Arbeitsgestaltung nach Eberhard Ulich (1978) sieht vor, Technik am Arbeitsplatz so zu gestalten, dass die Aufgaben für Menschen mit unterschiedlichen Fähigkeiten und Leistungsvoraussetzung erfüllt werden können. Industrielle Arbeitsumgebungen müssen damit den Menschen, die Unternehmensorganisation sowie die Technik gemeinsam betrachten und in ihren wechselseitigen Abhängigkeiten in die Technikgestaltung miteinbeziehen (Hartmann 2015). Zu starre Technologien führen dazu, dass Mitarbeiter:innen ,in ihren Fähigkeiten , eingefroren werden und keine Entwicklungsanreize erkennen können“. (Hartmann 2015, S. 12).

Diese Erkenntnisse sind mit Blick auf die Gestaltung digital-vernetzter Arbeitsumgebungen wieder zu aktualisieren. Gerade im Werkzeugmaschinenbau werden CNC-Steuerungen künftig auch auf intelligenten und damit noch komplexeren und intransparenteren Programmen basieren. Darum muss neben der intuitiven und einfachen Nutzbarkeit auch eine informierte und nachvollziehbare Nutzbarkeit bei der Entwicklung von Mensch-Maschine-Schnittstellen im Fokus stehen. Um der zunehmenden Verblackboxung komplexer Systeme im (Arbeits-)Alltag zu entgehen, müssen Benutzeroberflächen zum Teil komplett neu gedacht werden. Eine solche lernförderliche Arbeitsgestaltung ist eine Innovation an sich und dient der Innovationsfähigkeit der anwendenden Unternehmen, sodass sich mit ihr ein Wettbewerbsvorteil erzielen lässt.

Sowohl durch intransparentes Technikdesign als auch durch mangelnde Schulungen können Fehlentscheidungen passieren und auch die Möglichkeit, Verantwortung für bestimmte Produktionsprozesse zu übernehmen, wird in beiden Fällen gehemmt. Dies kann betriebsschädigende Auswirkungen haben (SVRV 2017). Für Schulungen empfehlen die befragten Expert:innen, das Interesse und die Eigeninitiative der Fachkräfte ins Zentrum zu rücken. Das würde auch bedeuten, Weiterbildungen ,,just in time" dort anzusetzen, wo ein spezifisches Thema oder Problem am individuellen Arbeitsplatz auftaucht. Schulungen würden dadurch in kürzeren Abständen und anhand konkreter Beispiele des eigenen Arbeitslebens stattfinden.

Interdisziplinäres Lernen und die Verknüpfung unterschiedlicher Fachdisziplinen ist ein weiterer Aspekt, der von den Expert:innen als für die Digitalisierung zukunftsrelevant genannt wird. Neue Ausbildungsprofile werden erforderlich, die betriebswirtschaftliche, rechtliche, soziale und technische Phänomene neu zusammenbringen. Ähnliche Erkenntnisse zeigt auch die Studie von Jürgen Dispan auf, der von einem Bedarf „an interdisziplinärer Intelligenz” (Dispan 2018, S. 12) spricht. Um die Weiterbildung als wesentlichen Bestandteil digitaler Unternehmensstrategien zu verankern, schlägt Dispan (2017) zudem vor, bei der Einbindung digitaler Technologie „Betriebsräte frühzeitig ein(zu)schalten, die Interessen der Belegschaft einbringen 
und den Prozess kritisch begleiten.“ (Dispan 2017, S. 28) Eine Beteiligung der Mitarbeiter:innen bei der Einführung digitaler Technologien erscheint sinnvoll, um sie selbst dazu zu befähigen, die Veränderungen für sich oder den daraus folgenden Qualifikationsbedarf besser einschätzen zu können (sowie auch vom Arbeitgeber einzufordern).

\subsection{Individualisierung der KI-basierten Technologien: Bedarf an Erprobungsszenarien}

Die befragten Expert:innen bemerkten eine Unsicherheit seitens der KMU im Werkzeugmaschinenbau darüber, was sie im Einzelnen von neueren digitalen Technologien wie KI- oder Cloud-Diensten erwarten können. Dies korreliert mit den Erfahrungen, die zur Implementierung verschiedener Innovationsprogramme des Bundes und der Länder führte (Demary et al. 2016; BMWi 2020b). Es besteht ein hoher Bedarf an Erprobungsszenarien, um neue digitale Technologien kennenzulernen und ihre Passung bzw. Anpassungsfähigkeit an die Bedarfe des eigenen Unternehmens auszuprobieren. Erst über Erprobungsszenarien lässt sich das Potenzial für die eigenen Arbeitsprozesse realistisch einschätzen, noch bevor große Investitionen getätigt werden. Dies betrifft Prozesse der Produkterstellung, der Produktionsorganisation und -durchführung, der Qualitätssicherung sowie der Erweiterung des bestehenden Produkt- und Serviceportfolios. Gleichzeitig kann von der Erprobung verschiedener Unternehmen auch die Technikgestaltung profitieren, da komplexes Erfahrungswissen aus den einzelnen Anwendungsgebieten in der Technikgestaltung berücksichtigt werden kann und Bedarfe konkret reflektiert werden können. Auch eine Studie der Plattform Lernende Systeme sieht in der stärkeren Einbeziehung der Beschäftigten in die Technikgestaltung große Potenziale. Bezogen auf das Transformationsmanagement von Unternehmen für die Einbeziehung von KI-Systemen lautet die Einschätzung, dass es „,nicht nur um das ,Mitnehmen der Menschen“ (gehe), sondern auch darum, die Beschäftigten dazu zu befähigen, dass sie selbstbewusst und kompetent mit KI-Systemen umgehen und deren Einführung mit ihrer Erfahrung und ihrem Wissen proaktiv mitgestalten können.“ (Plattform lernende Systeme 2019, S. 3)

Diese stärkere Einbeziehung könnte auch die nach Meinung der befragten Expert:innen bestehende Problematik verbessern, dass undurchsichtige Benutzerschnittstellen ein Hindernis in der Einbindung digitaler Technologien darstellen. Gerade in der Bedienung komplexer Maschinen entsteht umfangreiches Fachwissen, das nur schwer ohne die Beteiligung der Belegschaft in der Technikkonzeption $\mathrm{zu}$ berücksichtigen ist. Schon allein zur Einbindung verschiedener Produktionsmaschinen und zur Verknüpfung von Maschinen ist eine langjährige Erfahrung in der praktischen Interaktion mit einem bestimmten Maschinenpark nötig. Die aktuell großskalig angelegten Digitalisierungsangebote zielen auf die Nutzbarkeit eines möglichst großen Anwenderkreises und berücksichtigen nicht das spezielle Erfahrungswissen von Werker:innen im Werkzeugmaschinenbau.

Heute verfügbare digitale Technologien sind häufig zu intransparent, um sie in den Produktentstehungsprozess und in das bestehende Verantwortungsgefüge fachkräftebasierter, mittelständischer Unternehmen kontrolliert einbinden zu können. Dies betrifft insbesondere die Einbindung von KI-Technologien. Ein Bericht des BMWI 
zum Stand der KI-Nutzung in Deutschland stellt fest, dass nur $16 \%$ der KI einsetzenden Unternehmen ihre KI-Anwendungen selbst entwickeln. „In $24 \%$ erfolgte die Entwicklung sowohl durch das Unternehmen selbst als auch durch Dritte. $60 \%$ griffen auf KI-Entwicklungen durch Dritte zurück“ (BMWi 2020a). Auch im Werkzeugmaschinenbau könnten KI-Technologien in Zukunft Anwendung finden: Beispielsweise könnten bei der Aufgabe, komplexe Geometrien zu fräsen, KI-basierte Programme Handlungsempfehlungen auf Basis früherer Frässtrategien geben. Mit maschinellen Lernverfahren würde das System eigenständig Muster in vorherigen Datensätzen (sogenannte Lern- oder historische Daten) erkennen und den Werker:innen Lösungswege empfehlen. Doch es ist eine große Herausforderung, gerade adaptive (selbstlernende) Systeme zu konzipieren, die sich für hochqualifizierte Fachkräfte, wie sie im Werkzeugmaschinenbau tätig sind, erklärbar machen. Für die Frage, wie ein neuartiges Design der Mensch-Maschine-Schnittstelle aussehen könnte, das digital-souveräne Nutzer:innen mit eigenem Erfahrungswissen und individuellen Problemlösungskonzepten mitdenkt, gibt es aktuell nur sehr wenige Vorbilder und kaum erprobtes Technikdesign. Anstatt der Werkzeugmechaniker:in nur eine Fertigungsstrategie als die beste $\mathrm{zu}$ empfehlen, ohne aufzuzeigen, auf welcher Datenbasis und auf welchen Annahmen, Parametern oder anderen technischen Rahmenbedingungen diese Empfehlung beruht, muss das System in diesem Anwendungsfall für die Fachkraft nachvollziehbar und plausibel bleiben. Die angebotenen Handlungsempfehlungen sollten verständlich und handhabbar sein, um für die jeweilige Situation eine Lösung begründet wählen zu können. Weiterhin sollten die Anwendenden die Möglichkeit haben, bestimmte Annahmen und Erfahrungen, die sich als erfolgreich erwiesen haben, zu priorisieren. Eine undurchsichtige Handlungsempfehlung würde letztlich nicht nur die digitale Souveränität der Facharbeiter:innen einschränken, sondern auch das Vertrauen in das betreffende digitale Unterstützungsangebot mindern.

\section{Exkurs 2: Gestaltungsprinzip ,Erklärbare KI“6}

Maschinelles Lernen (ML) ist eine aktuelle KI-Anwendung, die aufgrund ihrer unüberschaubaren Größe an Daten und Verarbeitungsschritten in der Anwendung im Alltag schwer nachvollziehbar ist. Auch die inneren Logiken und Entscheidungsmechanismen sind für die Nutzenden intransparent oder unplausibel und damit nicht ohne Weiteres verantwortungsvoll in bestehende Produktionsprozesse einzubeziehen. Den Anwenderinnen und Anwendern einen ,digitalsouveränen“"Einsatz von ML-Algorithmen im Alltag zu ermöglichen, ist darum eine große Herausforderung in der aktuellen Forschung.

Maschinelles Lernen bietet sich auch im Werkzeugmaschinenbau künftig als hilfreiches Instrument an, etwa um zu verarbeitendes Rohmaterial vor der Bearbeitung auf Beschädigungen $\mathrm{zu}$ prüfen. Für einen verantwortungsvollen und effizienten Einsatz der Technologie muss die bedienende Fachkraft jedoch ungefähr verstehen, woran die Software beispielsweise Kratzer und Beulen als Beschädigungen im Material erkannt hat, um eine menschliche Prüfung zu 
ermöglichen und bei Fehlern eingreifen zu können. Um den Nutzer:innen solche Informationen zu gewährleisten, muss sie als ,digital-souverän“ bereits in der technischen Konzeption der ML-Algorithmen von Anfang an mitgedacht werden. Aktuell herrscht dazu ein hoher Forschungsbedarf und es werden erste Methoden zu ,erklärbarer KI“ entwickelt (Molnar 2019, Plattform Lernende Systeme 2020, S. 14/15). Bei „erklärbarer KI“ handelt es sich um Verfahren, mit denen den Nutzer:innen Basisinformationen über die Berechnungen zurückgegeben werden. Dem Nutzer werden dabei innere Logiken und Funktionsweisen transparent gemacht, z. B. nach welchen Parametern, Kategorien oder Annahmen die Algorithmen beschädigte Materialien identifiziert, bewertet und beurteilt haben. Ein Ansatz dafür ist die ,contrafaktische Erklärung“ (Wachter et al. 2018). Hier werden Entscheidungsgrenzen offengelegt, an denen die maschinelle Entscheidung umschlägt (z. B. „,das ist fehlerhaftes Material“/,das ist kein fehlerhaftes Material“). Ein anderes Vorgehen, Erklärungen zu maschinellen Lernverfahren zu erstellen, schlagen Schaaf et al. (2019) vor: Neuronale Netze können nachträglich durch Entscheidungsbäume approximiert werden, um die Logiken des neuronalen Netzes nachvollziehbar zu machen.

Gerade für den Anwendungsfall, dass informierte Nutzer:innen bzw. „digital-souveräne“ Fachkräfte mit KI interagieren, könnten Methoden der „,erklärbaren KI“ von Bedeutung sein (vgl. Exkurs 2, Gestaltungsprinzip „Erklärbare KI“). Erklärbare KI setzt an der Schnittstelle zwischen digitalen Technologien und dem Kompetenzerwerb der Fachkräfte an. Dies ist eine Möglichkeit, dass digitale Technologien bei KMU „digital souverän“ angewendet werden können. Auch die Lernförderlichkeit, wie sie im Exkurs 1 dargestellt wurde, wird hier in gewisser Weise berücksichtigt. Dieses Gestaltungsprinzip digitaler Technologien kann auf unterschiedliche Produktionsschritten angewendet werden - darunter z. B. Konstruktion, Simulation, Produktionsplanung, Wartung/Service, Bildung/Assistenz.

Im Werkzeugmaschinenbau ist die individuelle Anpassung und die Erklärbarkeit von Digitalisierungsstrategien in besonderer Weise nötig, da häufig maßgeschneiderte und individuelle Kundenanforderungen bearbeitet werden (Losgröße 1 bzw. sehr kleine Serien). Unabhängig voneinander kommen die Expert:innen zu dem Schluss, dass gerade hier die Digitalisierung im Sinne von „one size fits all“ nicht funktioniert. Unternehmen benötigen vielmehr die Möglichkeit zur individuellen Adaption ihrer Software und ihrer genutzten digitalen Dienste. Auch die Frage nach der Verantwortung von Datenzugängen, Datenspeicherung und -weitergabe bezüglich des Datenschutzes und der Datenzugänge wird hierbei relevant.

\subsection{Bedeutung und Weiterentwicklung der Trends für die Digitale Souveränität für KMU}

Entlang der Analyse in 2.2 und 2.3 konnten folgende Entwicklungspotenziale für KMU identifiziert werden. Zuvorderst besteht die Notwendigkeit, auch kleineren Unternehmen bestimmte Erprobungszenarien neuer digitaler Systeme (wie zum 
Beispiel KI) zu ermöglichen. Die Frage stellt sich, wie skalierbare Softwareangebote kostengünstig auf individuelle Bedarfe kleinerer Unternehmen angepasst werden können. Wenn KMU diese Erprobungsmöglichkeiten hätten, böten sich weiterführende Innovationspotenziale wie die Individualisierung digitaler Technologien und mit ihr die Gestaltung alternativer Bedienoberflächen, die gerade für hochqualifizierte Fachkräfte souveräne Umgangsweisen gewährleisten könnten. Vor allem im Werkzeugmaschinenbau verfügen die Mitarbeiter:innen über wichtiges Know-How im Produktionsprozess, dass nur über nachvollziehbare Nutzerführungen miteinbezogen werden kann. Diese Tendenz der Individualisierung von Software kulminiert in der aktuellen Forschung zu ,erklärbarer KI“, mit der neue digitale Systeme veantwortungsbewusst und individuell in Produktionsprozesse miteinbezogen werden können. Wie eine solche Gestaltung erklärbarer KI konkret aussehen könnte, gilt es zukünftig zu erforschen.

Neben dem Mangel an passgenauen Digitalisierungsangeboten für Fachkräfte und KMU stellen die Expert:innen auch einen Mangel an Schulungsangeboten zur Digitalisierung in den Betrieben fest. Wendet man diese Hemmnisse derzeitiger Digitalisierung zu Chancen alternativer Digitalisierungsangebote (siehe Tab. 1), so können mit einer behutsamen und maßgeschneiderten Digitalisierung Softwareprodukte entwickelt werden, die die digitale Souveränität von KMU und ihren hochqualifizierten Fachkräften ernst nehmen. Gestaltungsprinzipien für digitale Lösungen, die eine souveräne Nutzung ermöglichen, könnten ein Alleinstellungsmerkmal im Werkzeugmaschinenbau sein und damit als künftiger Wettbewerbsvorteil fungieren.

Eine solche Neuauflage digitaler Produkte, die die digitale Souveränität von KMU in den Fokus rückt, geht einerseits mit der Entwicklung neuer Kompetenzen von Fachkräften durch neue Schulungskonzepte einher. Andererseits bedarf es Erprobungsszenarien digitaler Technologien wie etwa KI-basierte Assistenzsysteme, um informierte Entscheidungen zur Einbindung digitaler Systeme ins Unternehmen treffen zu können. Die Frage nach einem digital-souveränen Umgang betrifft nicht nur KI-Systeme. Auch für Cloud-Systeme sind neue Konzepte für einen verantwortungsvollen Umgang mit Unternehmensdaten notwendig, um den hohen Ansprüchen der Unternehmen mit exzellentem Datenschutz gerecht zu werden. Transparenz und Nachvollziehbarkeit zur Ermöglichung eines digital-souveränen Umgangs im Arbeitsalltag gelten auch an anderen Stellen als wesentliche Gestaltungsprinzipien, die von vornherein in der technischen Konzeption bedacht werden müssen.

\section{Ausblick: Werkzeugmaschinenbau als Modell für andere Wirtschaftsbereiche}

Der Werkzeugmaschinenbau steht seit vielen Jahrzehnten für das Gestaltungsprinzip der Souveränität von Menschen im Umgang mit Maschinen ein. Hochqualifiziertes Fachpersonal wird in diesem Bereich stetig an hochtechnisierten Maschinen fortgebildet. Diese Kombination erzeugt den großen Erfolgsfaktor deutscher Unternehmen in diesem Bereich, dass Hightech mit hoher Flexibilität angeboten werden kann. 
Bringt man diese grundsätzliche Unternehmenskultur des Werkzeugmaschinenbaus - Fachkräfte stetig entlang komplexer Technologien weiter zu schulen - mit den oben skizzierten Digitalisierungstrends zusammen, so ergibt sich insbesondere in dieser Branche die Chance, Technikgestaltung und Schulungsangebote Hand in Hand und damit digital souverän voranzutreiben. Wie eine solche Umsetzung gelingen kann, lässt sich damit am Werkzeugmaschinenbau näher analysieren, Unternehmen können bei der Einbindung digitaler Technologien begleitet und ihre Erfahrungen auch auf andere Wirtschaftsbereiche übertragen werden. Folgende weiterführende Forschungsfragen leiten sich daraus ab:

- Welche Art der Technikgestaltung und Fachkräfteschulung ist in spezifischen Produktionsprozessen erforderlich?

- Wie erfolgt die Auslagerung von Maschinendaten in herstellergebundene Clouds oder die Einbindung von intransparenten, adaptiven KI-Systemen in den Arbeitsprozess?

- Welche Gestaltungsmöglichkeiten gibt es, um die digitale Souveränität des Menschen an der Maschine zu gewährleisten?

Die Branche des Werkzeugmaschinenbaus kann beispielsweise für Digitalisierungsstrategien einstehen, mit denen Lock-in-Effekte großskaliger Digitalisierungsangebote für KMU vermieden werden können. Zudem verfügt diese Branche über gute Fachkräfte, die - entsprechend geschult - auch eine Vorbildfunktion für den „digital souveränen“ Umgang mit neuen Technologien erfüllen können. Auch in der Technikgestaltung bieten sich zahlreiche Innovationspotenziale: Damit neuartige digitale Technologien einen Facharbeiter nicht in seiner Handlungsfreiheit und Kreativität einschränken, muss die Einbettung von KI-basierten Assistenzsystemen oder Cloud-Diensten nachvollziehbar, situativ und hinterfragbar bleiben. Bei der Digitalisierung souverän zu bleiben heißt damit, für den Werkzeugmaschinenbau mit Fokus auf die dort tätigen Facharbeiter:innen zwei Ebenen neu zu gestalten: Für die Hersteller bedeutet es, Technologien so zu entwickeln, dass die Anwendenden samt ihrer individuellen Kompetenzen und Erfahrungen in den Mittelpunkt gestellt werden (siehe 2.3). Gleichzeitig ergibt sich für die anwendenden Unternehmen dieser nutzerzentrierten Technologien der Auftrag, innerbetriebliche Organisationskonzepte so zu konzipieren, dass sie die Lernförderlichkeit der neuen Technologien bestmöglich unterstützen (etwa mit Schulungskonzepten, siehe 2.2). Mit diesem Gestaltungsprinzip - die digitale Souveränität und damit die Kompetenzen der Menschen ernst zu nehmen - lassen sich künftig wichtige Alleinstellungsmerkmale für die Produkte mittelständisch geprägter Maschinenbauer erzeugen. Neuartige digitale, effiziente und wettbewerbsfähige Techniksysteme können im Werkzeugmaschinenbau entstehen, wenn digitale Souveränität als ein solches Grundprinzip in die Technikgestaltung miteinfließt. 


\section{Literatur}

Blum, U., Hartmann, E. A.: Facharbeitsorientierte CNC-Steuerungs- und -Vernetzungskonzepte, S. 441-446. Werkstatt und Betrieb (1988)

BMWi: Digitale Souveränität und Künstliche Intelligenz - Voraussetzungen, Verantwortlichkeiten und Handlungsempfehlungen. Nürnberg (2018)

BMWi: Einsatz von Künstlicher Intelligenz in der Deutschen Wirtschaft. Stand der KI-Nutzung im Jahr 2019 (2020a). https://www.bmwi.de/Redaktion/DE/Publikationen/Wirtschaft/einsatz-von-ki-deutsche-wirtschaft.pdf?_blob=publicationFile \&v $=808.07 .2020$

BMWi: Digitalisierung im Mittelstand voranbringen (2020b). https://www.bmwi.de/Redaktion/ DE/Dossier/mittelstand-digitalisieren.html. Zugegriffen: 20. Juli 2020

Demary, V., Engels, B., Röhl, K.H., Rusche, C.: Digitalisierung und Mittelstand (IW-Analysen Nr. 109). Köln (2016)

Dispan, J.: Entwicklungstrends im Werkzeugmaschinenbau 2017. Kurzstudie zu Branchentrends auf Basis einer Literaturrecherche. Working Paper Forschungsförderung Nr. 029. Hans Böckler Stiftung (2017)

Dispan, J.: Digitale Transformation im Werkzeugmaschinenbau. Momentaufnahme zu Strategien, Stand und Wirkung der Digitalisierung (Industrie + Energie). IG Metall, Frankfurt a. M. (2018)

Dispan, J., Schwarz-Kocher, M.: Industrie 4.0. Maschinenbau: Wie Digitalisierung gelingt. Böckler Impuls, 4, 6-7 (2019)

Ensthaler, J., Haase, M.S.: Datenhoheit und Datenschutz im Zusammenhang mit Smart Services: Begleitforschung Smart Service Welt I (2017)

Hartmann, E.: Arbeitsgestaltung für Industrie 4.0: Alte Wahrheiten, neue Herausforderungen. In: Botthof, A., Hartmann, E.A. (Hrsg.) Zukunft der Arbeit in Industrie 4.0, S. 9-20. Springer, Heidelberg (2015). https://doi.org/10.1007/978-3-662-45915-7_2

Künzel, M., Kraus, T., Straub, S.: Begleitforschung PAiCE und iit, Förder- und Technologieprogramm des Bundesministeriums für Wirtschaft und Energie Platforms/Kollaboratives Engineering: Grundzüge und Herausforderungen der unternehmensübergreifenden Zusammenarbeit beim Engineering von Produkten und begleitenden Services (2019). https://www.digitale-technologien.de/DT/Redaktion/DE/Downloads/Publikation/2019-0401-paice-studie-engineering.pdf?_blob=publicationFile\&v=2. Zugegriffen: 19. Dez. 2019

Molnar, C.: Interpretable machine learning. A Guide for making black box models explainable (2019). https://christophm.github.io/interpretable-ml-book/index.html. Zugegriffen: 11. Dez. 2019

Schaaf, N., Huber, M., Maucher J.: Enhancing Decision Tree Based Interpretation of Deep Neuronal Networks through L1-Orthogonal Regularization. IEEE International Conference on Machine Learning and Applications (ICMLA), S. 42-49 (2019)

PAiCe - Seifert, I., Bürger, M. Wangler, L., Christman-Budian, S., Rohde, M., Gabriel, P., Zinke, G.: Begleitforschung PAiCE und iit, Förder- und Technologieprogramm des Bundesministeriums für Wirtschaft und Energie Platforms. Potenziale der Künstlichen Intelligenz im produzierenden Gewerbe in Deutschland (2018). https://www.bmwi.de/Redaktion/DE/ Publikationen/Studien/potenziale-kuenstlichen-intelligenz-im-produzierenden-gewerbe-indeutschland.pdf?_blob=publicationFile\&v=8. Zugegriffen: 10. Nov. 2019

Plattform Lernende Systeme. (2020). https://www.plattform-lernende-systeme.de/files/Downloads/Publikationen/AG2_Whitepaper2_220620.pdf. Zugegriffen: 31. Juli 2020 
Plattform Lernende Systeme. (2019). https://www.plattform-lernende-systeme.de/files/Downloads/Publikationen/AG2_Whitepaper_210619.pdf. Zugegriffen: 31. Juli 2020

Stubbe, J., Schaat, S., Ehrenberg-Silies, S.: Digital souverän?. Kompetenzen für ein selbstbestimmtes Leben im Alter. Bertelsmann Stiftung, Güterloh (2019)

SVRV: Digitale Souveränität. Gutachten des Sachverständigenrats für Verbraucherfragen (2017)

Ulich, E.: Über das Prinzip der differentiellen Arbeitsgestaltung. Industrielle Organisation 47, $566-568$ (1978)

Wachter, S., Mittelstadt, B., Russel, C.: Counterfactual Explanations without opening the Black Box. Automated Decisions and the GDPR (2018)

Wittpahl, V. (Hrsg.): Digitale Souveränität. Bürger, Unternehmen, Staat. Springer Vieweg Open, Berlin (2017)

Open Access Dieses Kapitel wird unter der Creative Commons Namensnennung 4.0 International Lizenz (http://creativecommons.org/licenses/by/4.0/deed.de) veröffentlicht, welche die Nutzung, Vervielfältigung, Bearbeitung, Verbreitung und Wiedergabe in jeglichem Medium und Format erlaubt, sofern Sie den/die ursprünglichen Autor(en) und die Quelle ordnungsgemäß nennen, einen Link zur Creative Commons Lizenz beifügen und angeben, ob Änderungen vorgenommen wurden.

Die in diesem Kapitel enthaltenen Bilder und sonstiges Drittmaterial unterliegen ebenfalls der genannten Creative Commons Lizenz, sofern sich aus der Abbildungslegende nichts anderes ergibt. Sofern das betreffende Material nicht unter der genannten Creative Commons Lizenz steht und die betreffende Handlung nicht nach gesetzlichen Vorschriften erlaubt ist, ist für die oben aufgeführten Weiterverwendungen des Materials die Einwilligung des jeweiligen Rechteinhabers einzuholen.

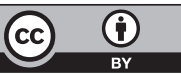

